Journal of Applied Mathematics and Stochastic Analysis, 16:4 (2003), 295-309.

Printed in the USA (C)2003 by North Atlantic Science Publishing Company

\title{
BACKWARD STOCHASTIC DIFFERENTIAL EQUATIONS WITH OBLIQUE REFLECTION AND LOCAL LIPSCHITZ DRIFT
}

\author{
AUGUSTE AMAN and MODESTE N'ZI \\ URF de Mathématiques et Informatique \\ 22 BP 582 Abidjan 22, Côte d'Ivoire
}

(Received January, 2003; Revised September, 2003)

\begin{abstract}
We consider reflected backward stochastic differential equations with time and space dependent coefficients in an orthant, and with oblique reflection. Existence and uniqueness of solution are established assuming local Lipschitz continuity of the drift, Lipschitz continuity and uniform spectral radius conditions on the reflection matrix.
\end{abstract}

Keywords: Backward Stochastic Differential Equations, Oblique Reflection, Brownian Motion.

AMS (MOS) subject classification: 60H10, 60H20.

\section{Introduction}

It was mainly during the last decade that the theory of backward stochastic differential equations took shape as a distinct mathematical discipline. This theory has found a wide field of applications as in stochastic optimal control and stochastic games (see Hamadène and Lepeltier [9]), in mathematical finance via the theory of hedging and nonlinear pricing theory for imperfect markets (see El Karoui et al.[6]). Backward stochastic differential equations also appear to be a powerful tool for constructing $\Gamma$-martingales on manifolds (see Darling [4]). These kind of equations provide probabilistic formulae for solutions to partial differential equations (see Pardoux and Peng [14]).

Consider the following linear backward stochastic differential equation

$$
\left\{\begin{aligned}
-d Y_{s} & =\left[Y_{s} \beta_{s}+Z_{s} \gamma_{s}\right] d s-Z_{s} d B_{s}, 0 \leq s \leq T \\
Y_{T} & =\xi
\end{aligned}\right.
$$

As is well known, equation (1.1) was first introduced by Bismut $[1,2]$ when he was studying the adjoint equation associated with the stochastic maximum principle in optimal stochastic control. It is used in the context of mathematical finance as the model behind the Black and Scholes formula for the pricing and hedging option. 
The development of general backward stochastic differential equation (BSDE in short)

$$
\left\{\begin{aligned}
-d Y_{s} & =f\left(s, Y_{s}, Z_{s}\right) d s-Z_{s} d B_{s}, 0 \leq s \leq T \\
Y_{T} & =\xi
\end{aligned}\right.
$$

begins with the paper of Pardoux and Peng [14]. Since then, BSDEs have been intensively studied. For example, BSDE with reflecting barrier have been studied among others by El Karoui et al. [5], Cvitanic and Karatzas [3], Matoussi [12] and Hamadène et al.[10] in the one dimensional case. The higher dimensional one has been considered by Gegout-Petit and Pardoux [8] for reflection in a convex domain. The multivalued context can be found in Pardoux and Rascanu [15], N'zi and Ouknine [13], Hamadène and Ouknine [11] and Essaky et al [7].

These works concern the case of normal reflection at the boundary. In the last two decades, thanks to the numerous applications in queuing theory, the deterministic as well as stochastic Skorokhod problem (in a convex polyhedron with oblique reflection at the boundary) has been studied by many authors. Recently, S. Ramasubramanian [16] has considered reflected backward stochastic differential equations (RBSDE's) in an orthant with oblique reflection at the boundary. He has established the existence and uniqueness of the solution under a uniform spectral radius condition on the reflection matrix (plus of course, a Lipschitz continuity condition on the coefficient).

The aim of this article is to weaken the Lipschitz condition on the drift to a locally Lipchitz one. The paper is organized as follows. In section 2 , we introduce the underlying assumptions and state the main result. Section 3 is devoted to the proof of the main result.

\section{Assumptions and Formulation of the Main Result}

Let $B=\left\{B(t)=\left(B_{1}(t), \ldots, B_{d}(t)\right): t \geq 0\right\}$ be a $d$ - dimensional standard Brownian motion defined on a probability space $(\Omega, \mathcal{F}, P)$ and let $\left\{\mathcal{F}_{t}\right\}$ be the natural filtration generated by $B$, with $\mathcal{F}_{0}$ containing all $P$-null sets.

Let $G=\left\{x \in \mathbb{R}^{d}: x_{i}>0,1 \leq i \leq d\right\}$ denote the $d$-dimensional positive orthant.

We are given the following:

- $T>0$ is a terminal time;

- $\xi$ is an $\mathcal{F}_{T}$-measurable, bounded, $\bar{G}$-valued random variable;

- $b:[0 ; T] \times \Omega \times \mathbb{R}^{d} \longrightarrow \mathbb{R}^{d}, R:[0 ; T] \times \Omega \times \mathbb{R}^{d} \longrightarrow \mathbb{M}_{d}(\mathbb{R})$ are both bounded measurable functions such that for every $y \in \mathbb{R}^{d}, b(., ., y)=\left(b_{1}(., ., y), \ldots, b_{d}(., ., y)\right)$ and $R(., ., y)=\left(r_{i j}(., ., y)\right)_{1 \leq i, j \leq d}$ are $\mathcal{F}_{t}$-predictable processes. We also assume that $r_{i i}(., .,.) \equiv 1$. (Here $\mathbb{M}_{d}(\mathbb{R})$ denotes the class of $d \times d$ matrices with real entries).

Definition 2.1: A triple $Y=\left\{Y(t)=\left(Y_{1}(t), . ., Y_{d}(t)\right): t \geq 0\right\} ; Z=\{Z(t)=$ $\left.\left(Z_{i j}(t)\right)_{1 \leq i, j \leq d}: t \geq 0\right\}$ and $K=\left\{K(t)=\left(K_{1}(t), . ., K_{d}(t)\right): t \geq 0\right\}$ of $\left\{\mathcal{F}_{t}\right\}$-progressively measurable integrable processes is said to solve $\operatorname{RBSDE}(\xi, b, R)$ if the following hold:

(i) $(Y, Z, K)$ is a continuous $\mathbb{R}^{d} \times \mathbb{M}_{d}(\mathbb{R}) \times \mathbb{R}^{d}$-valued process; 
(ii) for every $i=1, \ldots, d$, and $0 \leq t \leq T$,

$$
\begin{aligned}
Y_{i}(t)= & \xi_{i}+\int_{t}^{T} b_{i}(s, Y(s)) d s-\sum_{j=1}^{d} \int_{t}^{T} Z_{i j}(s) d B_{j}+K_{i}(T)-K_{i}(t) \\
& +\sum_{j \neq i} \int_{t}^{T} r_{i j}(s, Y(s)) d K_{j}(s)
\end{aligned}
$$

(iii) for every $0 \leq t \leq T, \quad Y(t) \in \bar{G}$;

(iv) for every $1 \leq i \leq d, K_{i}(0)=0, K_{i}(\cdot)$ is nondecreasing and can increase only when $Y_{i}(\cdot)=0$, that is

$$
K_{i}(t)=\int_{0}^{t} 1_{\{0\}}\left(Y_{i}(s)\right) d K_{i}(s) .
$$

We make the following assumptions on the coefficients $b, R$.

(A1) For every $1 \leq i \leq d, y \mapsto b_{i}(t, \omega, y)$ is locally Lipschitz continuous, uniformly over $(t, \omega)$; there is a constant $\beta_{i}$ such that $\left|b_{i}(t, \omega, y)\right| \leq \beta_{i}$, for all $(t, \omega, y) \in$ $[0 ; T] \times \Omega \times \mathbb{R}^{d}$.

(A2) For $1 \leq i, j \leq d, y \mapsto r_{i j}(t, \omega, y)$ is Lipschitz continuous, uniformly over $(t, \omega)$.

(A3) For every $i \neq j$ there exists constant $v_{i j}$ such that $\left|r_{i j}(t, \omega, y)\right| \leq v_{i j}$. Set $V=$ $\left(v_{i j}\right)$ with $v_{i i}=0$.We assume that $\sigma(V)<1$, where $\sigma(V)$ denotes the spectral radius of $V$. Therefore,

$$
(I-V)^{-1}=I+V+V^{2}+V^{3}+\cdots
$$

In the sequel, we put $\beta=\left(\beta_{1}, \ldots, \beta_{d}\right)$.

Remark 2.1: In view of (A3), there exists constants $a_{j}, 1 \leq j \leq d$ and $0<\alpha<1$ such that

$$
\sum_{i \neq j} a_{i}\left|r_{i j}(t, \omega, y)\right| \leq \sum_{i \neq j} a_{i} v_{i j} \leq \alpha a_{j}
$$

for all $j=1, \ldots, d$ and $(t, \omega, y) \in[0 ; T] \times \Omega \times \mathbb{R}^{d}$.

Let $\mathcal{H}$ stands for the space of all $\left\{\mathcal{F}_{t}\right\}$-progressively measurable, continuous pairs of processes $\left\{Y(t)=\left(Y_{1}(t), . ., Y_{d}(t)\right): t \geq 0\right\}$ and $\left\{K(t)=\left(K_{1}(t), . ., K_{d}(t)\right): t \geq 0\right\}$ such that

(i) for every $0 \leq t \leq T, Y(t) \in \bar{G}$;

(ii) for every $1 \leq i \leq d, K_{i}(0)=0 ; K_{i}(\cdot)$ is nondecreasing and can increase only when $Y_{i}(\cdot)=0$

(iii) $\mathbb{E}\left(\sum_{i=1}^{d} \int_{0}^{T} e^{\theta t} a_{i}\left|Y_{i}(t)\right| d t\right)<+\infty$;

(iv) $\mathbb{E}\left(\sum_{i=1}^{d} \int_{0}^{T} e^{\theta t} a_{i} \varphi_{t}\left(K_{i}\right) d t\right)<+\infty$; where $\varphi_{t}(g)$ denotes the total variation of $g$ over $[t, T]$ and $\theta>0$ is a fixed constant which will be chosen suitably later. 
For $(Y, K),(\widehat{Y}, \widehat{K}) \in \mathcal{H}$, we define the metric

$$
\begin{aligned}
d((Y, K),(\widehat{Y}, \widehat{K}))= & \mathbb{E}\left(\sum_{i=1}^{d} \int_{0}^{T} e^{\theta t} a_{i}\left|Y_{i}(t)-\widehat{Y}_{i}\right| d t\right) \\
& +\mathbb{E}\left(\sum_{i=1}^{d} \int_{0}^{T} e^{\theta t} a_{i} \varphi_{t}\left(K_{i}-\widehat{K}_{i}\right) d t\right) .
\end{aligned}
$$

It is not difficult to see that $(\mathcal{H}, d)$ is a complete metric space.

Let $\widetilde{\mathcal{H}}$ denote the collection of all $(Y, K) \in \mathcal{H}$ such that there exists an $\left\{\mathcal{F}_{t}\right\}$-progressively measurable process $\left\{D(t)=\left(D_{1}(t), \ldots . ., D_{d}(t)\right): t \geq 0\right\}$, with

$$
0 \leq D_{i}(t) \leq\left((I-V)^{-1} \beta\right)_{i} \text { a.s. and } K_{i}(t)=\int_{0}^{t} D_{i}(s) d s .
$$

Since $\widetilde{\mathcal{H}}$ is a closed subset of $\mathcal{H},(\widetilde{\mathcal{H}}, d)$ is a complete metric space.

We consider the norm $\|y\|=\sum a_{i}\left|y_{i}\right|$ which is equivalent to the Euclidean norm in $\mathbb{R}^{d}$. So, we may assume that the local Lipschitz continuity in (A1) and Lipschitz continuity in (A2) are with respect to this norm.

Before stating our main result, let us remark that if $(Y, K),(\widehat{Y}, \widehat{K}) \in \widetilde{\mathcal{H}}$ with $D_{i}, \widehat{D}_{i}$ being respectively the derivatives of $K_{i}, \widehat{K}_{i}$ then

$$
\varphi_{t}\left(K_{i}-\widehat{K}_{i}\right)=\int_{t}^{T}\left|D_{i}(s)-\widehat{D}_{i}(s)\right| d s .
$$

Therefore, using integration by parts in (2.1), we have

$$
\begin{aligned}
d((Y, K),(\widehat{Y}, \widehat{K}))= & \mathbb{E}\left(\sum_{i=1}^{d} \int_{0}^{T} e^{\theta t} a_{i}\left|Y_{i}(t)-\widehat{Y}_{i}\right| d t\right) \\
& +\mathbb{E}\left(\sum_{i=1}^{d} \int_{0}^{T} \frac{e^{\theta t}-1}{\theta} a_{i}\left|D_{i}(t)-\widehat{D}_{i}(t)\right| d t\right) \\
= & \mathbb{E}\left(\int_{0}^{T} e^{\theta t}|| Y(t)-\widehat{Y}(t) \| d t\right)+\mathbb{E}\left(\int_{0}^{T} \frac{e^{\theta t}-1}{\theta}\|D(t)-\widehat{D}(t)\| d t\right) .
\end{aligned}
$$

For every $z \in \mathbb{M}_{d}(\mathbb{R})$, we put

$$
|||z|||=\left(\sum_{j=1}^{d} \sum_{i=1}^{d} a_{i}\left|z_{i j}\right|^{2}\right)^{1 / 2} .
$$

Let $\mathbb{H}$ denote the space of all $\mathcal{F}_{t}$-progressively measurable processes $Z=\left(Z_{i j}\right)_{1 \leq i, j \leq d}$ such that

$$
\mathbb{E}\left(\int_{0}^{T}|\|Z(t)\||^{2} d t\right)<+\infty,
$$

endowed with the norm

$$
|Z|=\left[\mathbb{E}\left(\left.\int_{0}^{T}|\| Z(t)|\right|^{2} d t\right)\right]^{1 / 2}
$$


It is clear that $\mathbb{H}$ is a Banach space.

Now, we state our main result:

Theorem 2.1: Assume (A1)-(A3). Let $\xi$ be a bounded, $\mathcal{F}_{T}-$ measurable $\bar{G}$-valued random variable. Then there is a unique couple $((Y, K), Z) \in \widetilde{\mathcal{H}} \times \mathbb{H}$ solving $R B S D E$ $(\xi, b, R)$.

\section{Proof of the Main Result}

The proof of Theorem 2.1 needs some preliminary lemmas.

Lemma 3.1: Let $b$ be a process satisfying assumption $(\boldsymbol{A} 1)$. Then there exists a sequence of processes $b^{n}$ such that

(i) for each $n, b^{n}$ is Lipschitz continuous and $\left|b_{i}^{n}(t, \omega, y)\right| \leq \beta_{i}$, for all $1 \leq i \leq d$ and $(t, \omega, y) \in[0, T] \times \Omega \times \mathbb{R}^{d}$

(ii) for every $p, \rho_{p}\left(b^{n}-b\right) \rightarrow 0$ as $n \rightarrow+\infty$, where

$$
\rho_{p}(f)=\mathbb{E}\left(\int_{0}^{T} e^{\theta s} \sup _{|x|<p}\|f(s, x)\| d s\right) .
$$

Proof: Let $\psi_{n}$ be a sequence of smooth functions with support in the ball $B(0, n+1)$ such that $\sup \psi_{n}=1$. It not difficult to see that the sequence $\left(b^{n}\right)_{n \geq 1}$ of truncated functions defined by $b^{n}=b \psi_{n}$, satisfies all the properties quoted above.

In view of Ramasubramanian [16], there exists a unique couple of processes $\left\{\left(\left(Y^{n}(t)\right.\right.\right.$, $\left.\left.\left.K^{n}(t)\right), Z^{n}(t)\right): t \geq 0\right\} \in \widetilde{\mathcal{H}} \times \mathbb{H}$ solution to the $\operatorname{RBSDE}\left(\xi, b^{n}, R\right)$.

We formulate some uniform estimates for the processes $\left\{\left(\left(Y^{n}(t), K^{n}(t)\right), Z^{n}(t)\right): t \geq 0\right\}$ in the following way.

Lemma 3.2: Assume (A1)-(A3). Then there exists a constant $C$, such that for every $n \geq 1$

$$
\mathbb{E}\left(\int_{0}^{T} e^{\theta t}\left\|Y^{n}(t)\right\| d t\right)+\mathbb{E}\left(\int_{0}^{T} \frac{e^{\theta t}-1}{\theta}\left\|D^{n}(t)\right\| d t\right)<C .
$$

Proof:Let the triple $\left(Y^{n}, K^{n}, Z^{n}\right)$ be the unique solution of $\operatorname{RBSDE}\left(\xi, b^{n}, R\right)$. We have for every $i=1, \ldots, d$, and $0 \leq t \leq T$

$$
\begin{aligned}
Y^{n}(t)= & \xi_{i}+\int_{t}^{T} b_{i}^{n}\left(s, Y^{n}(s)\right) d s-\int_{t}^{T} \sum_{j=1}^{d} Z_{i j}^{n}(s) d B_{j}+K_{i}^{n}(T)-K_{i}^{n}(t) \\
& +\sum_{j \neq i} \int_{t}^{T} r_{i j}\left(s, Y^{n}(s)\right) d K_{j}(s) .
\end{aligned}
$$

Since

$$
\varphi_{t}\left(K_{i}^{n}\right)=\int_{t}^{T}\left|D_{i}^{n}(s)\right| d s
$$


applying Theorem $3.2[16]$ and using integration by parts, we obtain

$$
\begin{aligned}
& \mathbb{E}\left(\int_{0}^{T} \theta e^{\theta t}\left|Y_{i}^{n}(t)\right| d t\right)+\mathbb{E}\left(\int_{0}^{T} \theta e^{\theta t} \varphi_{t}\left(K_{i}^{n}\right) d t\right) \\
= & \mathbb{E}\left(\int_{0}^{T} \theta e^{\theta t}\left|Y_{i}^{n}(t)\right| d t\right)+\mathbb{E}\left(\int_{0}^{T}\left(e^{\theta t}-1\right)\left|D_{i}^{n}(t)\right| d t\right) \\
\leq & \mathbb{E}\left(\left(e^{\theta T}-1\right)\left|\xi_{i}\right|\right)+\mathbb{E}\left(\int_{0}^{T}\left(e^{\theta t}-1\right)\left|b_{i}^{n}\left(t, Y^{n}(t)\right)\right| d t\right) \\
+ & \mathbb{E}\left(\int_{0}^{T}\left(e^{\theta t}-1\right) \sum_{j \neq i}\left|r_{i j}\left(t, Y^{n}(t)\right)\right|\left|D_{j}^{n}(t)\right| d t\right) .
\end{aligned}
$$

We know that for every $(t, \omega, y)$ and every $i \neq j,\left|r_{i j}(t, \omega, y)\right| \leq v_{i j}$. Moreover for every $j=1, \ldots, d$ and $n \geq 1,\left|b_{j}^{n}(t, \omega, y)\right| \leq \beta_{j},\left|D_{j}^{n}(t, \omega, y)\right| \leq\left((I-V)^{-1} \beta\right)_{j}$.

Therefore

$$
\begin{array}{r}
\mathbb{E}\left(\int_{0}^{T} \theta e^{\theta t}\left|Y_{i}^{n}(t)\right| d t\right)+\mathbb{E}\left(\int_{0}^{T} \theta e^{\theta t} \varphi_{t}\left(K_{i}^{n}\right) d t\right) \\
\leq \mathbb{E}\left(\left(e^{\theta T}-1\right)\left|\xi_{i}\right|\right)+\mathbb{E}\left(\int_{0}^{T}\left(e^{\theta t}-1\right) \beta_{i} d t\right) \\
\quad+\mathbb{E}\left(\int_{0}^{T}\left(e^{\theta t}-1\right) \sum_{j \neq i} v_{i j}\left((I-V)^{-1} \beta\right)_{j} d t\right) .
\end{array}
$$

Let us note that

$$
d\left(\left(Y^{n}, K^{n}\right),(0,0)\right)=\mathbb{E}\left(\int_{0}^{T} e^{\theta t}\left\|Y^{n}(t)\right\| d t\right)+\mathbb{E}\left(\int_{0}^{T} \frac{e^{\theta t}-1}{\theta}\left\|D^{n}(t)\right\| d t\right) .
$$

Multiplying (3.2) by $a_{i}$ and adding leads to

$$
\begin{array}{r}
\theta d\left(\left(Y^{n}, K^{n}\right),(0,0)\right) \leq \mathbb{E}\left(\sum_{i=1}^{d}\left(e^{\theta T}-1\right) a_{i}\left|\xi_{i}\right|\right)+\mathbb{E}\left(\sum_{i=1}^{d} \int_{0}^{T}\left(e^{\theta t}-1\right) a_{i} \beta_{i} d t\right) \\
+\mathbb{E}\left(\int_{0}^{T}\left(e^{\theta t}-1\right) \sum_{i=1}^{d} \sum_{j \neq i} a_{i} v_{i j}\left((I-V)^{-1} \beta\right)_{j} d t\right) .
\end{array}
$$

In view of the inequality

$$
\sum_{i \neq j} v_{i j} a_{i} \leq \alpha a_{j}
$$


we have

$$
\begin{array}{r}
\theta d\left(\left(Y^{n}, K^{n}\right),(0,0)\right) \leq \mathbb{E}\left(\sum_{i=1}^{d}\left(e^{\theta T}-1\right) a_{i}\left|\xi_{i}\right|\right)+\mathbb{E}\left(\sum_{i=1}^{d} \int_{0}^{T}\left(e^{\theta t}-1\right) a_{i} \beta_{i} d t\right) \\
+\alpha \mathbb{E}\left(\int_{0}^{T}\left(e^{\theta t}-1\right) \sum_{j=1}^{d} a_{j}\left((I-V)^{-1} \beta\right)_{j} d t\right) \\
\leq\left(e^{\theta T}-1\right) \mathbb{E}\|\xi\| \\
+\left(\|\beta\|+\alpha\left\|\left((I-V)^{-1} \beta\right)\right\|\right) \int_{0}^{T}\left(e^{\theta t}-1\right) d t \\
\leq C .
\end{array}
$$

Hence inequality (3.1) is proved.

Now, we shall prove the convergence of the sequence $\left(Y^{n}, K^{n}, Z^{n}\right)_{n \geq 1}$.

Theorem 3.1: Assume (A1)-(A3). Then there exists $((Y, K), Z) \in \widetilde{\mathcal{H}} \times \mathbb{H}$ such that

$$
\lim _{n \rightarrow+\infty}\left\{\mathbb{E}\left(\int_{0}^{T} e^{\theta t}\left\|Y^{n}(t)-Y(t)\right\| d t\right)+\mathbb{E} \int_{0}^{T} \frac{e^{\theta t}-1}{\theta}\left\|D^{n}(t)-D(t)\right\| d t\right\}=0
$$

and

$$
\lim _{n \rightarrow+\infty} \mathbb{E}\left(\int_{0}^{T}\left\|Z^{n}(t)-Z(t)\right\| \|^{2} d t\right)=0
$$

where

$$
K_{i}(t)=\int_{0}^{t} D_{i}(s) d s, i=1, \ldots, d .
$$

Proof: It follows from the same idea used in the proof of inequality (3.1) that

$$
\begin{aligned}
& \mathbb{E}\left(\int_{0}^{T} \theta e^{\theta t}\left|Y_{i}^{m}(t)-Y_{i}^{n}(t)\right| d t\right)+\mathbb{E}\left(\int_{0}^{T} \theta e^{\theta t} \varphi_{t}\left(K_{i}^{m}-K_{i}^{n}\right) d t\right) \\
= & \mathbb{E}\left(\int_{0}^{T} \theta e^{\theta t}\left|Y_{i}^{m}(t)-Y_{i}^{n}(t)\right| d t\right)+\mathbb{E}\left(\int_{0}^{T}\left(e^{\theta t}-1\right)\left|D_{i}^{m}(t)-D_{i}^{n}(t)\right| d t\right) \\
\leq & \mathbb{E}\left(\int_{0}^{T}\left(e^{\theta t}-1\right)\left|b_{i}^{m}\left(t, Y^{m}(t)\right)-b_{i}^{n}\left(t, Y^{n}(t)\right)\right| d t\right) \\
& +\mathbb{E}\left(\int_{0}^{T}\left(e^{\theta t}-1\right) \mid \sum_{j \neq i} r_{i j}\left(t, Y^{m}(t)\right) D_{j}^{m}(t)-r_{i j}\left(t, Y^{n}(t) D_{j}^{n}(t) \mid d t\right)\right. \\
\leq & \mathbb{E}\left(\int_{0}^{T}\left(e^{\theta t}-1\right)\left|b_{i}^{m}\left(t, Y^{m}(t)\right)-b_{i}^{n}\left(t, Y^{n}(t)\right)\right| d t\right) \\
& +\mathbb{E}\left(\int_{0}^{T}\left(e^{\theta t}-1\right) \sum_{j \neq i} \mid r_{i j}\left(t, Y^{m}(t)-r_{i j}\left(t, Y^{n}(t)\right)|| D_{j}^{n}(t) \mid d t\right)\right.
\end{aligned}
$$




$$
+\mathbb{E}\left(\int_{0}^{T}\left(e^{\theta t}-1\right) \sum_{j \neq i}\left|r_{i j}\left(t, Y^{m}(t)\right)\right|\left|D_{j}^{m}(t)-D_{j}^{n}(t)\right| d t\right) .
$$

For an arbitrary number $N>1$, let $L_{N}$ be the Lipschitz constant of $b$ in the ball $B(0, N)$. We put

$$
A_{m, n}^{N}=\left\{\omega \in \Omega,\left\|Y^{m}(t, \omega)\right\|+\left\|Y^{n}(t, \omega)\right\|>N\right\}, \bar{A}_{m, n}^{N}=\Omega \backslash A_{m, n}^{N} .
$$

It follows that

$$
\begin{aligned}
& \mathbb{E}\left(\int_{0}^{T} \theta e^{\theta t}\left|Y_{i}^{m}(t)-Y_{i}^{n}(t)\right| d t\right)+\mathbb{E}\left(\int_{0}^{T}\left(e^{\theta t}-1\right)\left|D_{i}^{m}(t)-D_{i}^{n}(t)\right| d t\right) \\
\leq & \mathbb{E}\left(\int_{0}^{T}\left(e^{\theta t}-1\right)\left|b_{i}^{m}\left(t, Y^{m}(t)\right)-b_{i}^{n}\left(t, Y^{n}(t)\right)\right| 1_{A_{m, n}^{N}} d t\right) \\
+ & \mathbb{E}\left(\int_{0}^{T}\left(e^{\theta t}-1\right)\left|b_{i}^{m}\left(t, Y^{m}(t)\right)-b_{i}^{n}\left(t, Y^{n}(t)\right)\right| 1_{\bar{A}_{m, n}^{N}} d t\right) \\
+ & \mathbb{E}\left(\int_{0}^{T}\left(e^{\theta t}-1\right) \sum_{j \neq i} \mid r_{i j}\left(t, Y^{m}(t)-r_{i j}\left(t, Y^{n}(t)\right)|| D_{j}^{n}(t) \mid d t\right)\right. \\
+ & \mathbb{E}\left(\int_{0}^{T}\left(e^{\theta t}-1\right) \sum_{j \neq i}\left|r_{i j}\left(t, Y^{m}(t)\right)\right|\left|D_{j}^{m}(t)-D_{j}^{n}(t)\right| d t\right) \\
= & I_{1}+I_{2}+I_{3}+I_{4} .
\end{aligned}
$$

It not difficult to check that

$$
\begin{aligned}
I_{2}= & \mathbb{E}\left(\int_{0}^{T}\left(e^{\theta t}-1\right)\left|b_{i}^{m}\left(t, Y^{m}(t)\right)-b_{i}^{n}\left(t, Y^{n}(t)\right)\right| 1_{\bar{A}_{m, n}^{N}} d t\right) \\
\leq & \mathbb{E} \int_{0}^{T}\left(e^{\theta t}-1\right)\left|b_{i}^{m}\left(t, Y^{m}(t)\right)-b_{i}\left(t, Y^{m}(t)\right)\right| 1_{\bar{A}_{m, n}^{N}} d t \\
& +\mathbb{E}\left(\int_{0}^{T}\left(e^{\theta t}-1\right)\left|b_{i}\left(t, Y^{m}(t)\right)-b_{i}\left(t, Y^{n}(t)\right)\right| 1_{\bar{A}_{m, n}^{N}} d t\right) \\
& +\mathbb{E}\left(\int_{0}^{T}\left(e^{\theta t}-1\right)\left|b_{i}\left(t, Y^{n}(t)\right)-b_{i}^{n}\left(t, Y^{n}(t)\right)\right| 1_{\bar{A}_{m, n}^{N}} d t\right) .
\end{aligned}
$$


Since $b_{i}$ is $\frac{L_{N}}{a_{i}}$-locally Lipschitz, we get

$$
\begin{aligned}
I_{2} \leq & \mathbb{E}\left(\int_{0}^{T}\left(e^{\theta t}-1\right)\left|b_{i}^{m}\left(t, Y^{m}(t)\right)-b_{i}\left(t, Y^{m}(t)\right)\right| 1_{\bar{A}_{m, n}^{N}} d t\right) \\
& +\mathbb{E}\left(\int_{0}^{T}\left(e^{\theta t}-1\right)\left|b_{i}\left(t, Y^{n}(t)\right)-b_{i}^{n}\left(t, Y^{n}(t)\right)\right| 1_{\bar{A}_{m, n}^{N}} d t\right) \\
& +\frac{L_{N}}{a_{i}} \mathbb{E}\left(\int_{0}^{T}\left(e^{\theta t}-1\right)\left\|Y^{m}(t)-Y^{n}(t)\right\| d t\right) .
\end{aligned}
$$

In view of the Lipschitz condition on $R$ and the boundedness of $D_{j}^{n}(t)$, we obtain that there exists $C_{1}>0$ such that

$$
\begin{aligned}
I_{3} & \leq L \mathbb{E}\left(\int_{0}^{T}\left(e^{\theta t}-1\right)\left\|Y^{m}(t)-Y^{n}(t)\right\|\left|D_{j}^{n}(t)\right| d t\right) \\
& \leq L \mathbb{E}\left(\int_{0}^{T}\left(e^{\theta t}-1\right)\left\|Y^{m}(t)-Y^{n}(t)\right\| \sum_{j \neq i}\left((I-V)^{-1} \beta\right)_{j} d t\right) \\
& \leq L C_{1} \mathbb{E} \int_{0}^{T}\left(e^{\theta t}-1\right)\left\|Y^{m}(t)-Y^{n}(t)\right\| d t .
\end{aligned}
$$

Now, from the boundness of $R$, we have

$$
I_{4} \leq \mathbb{E}\left(\int_{0}^{T}\left(e^{\theta t}-1\right) \sum_{j \neq i} v_{i j}\left|D_{j}^{m}(t)-D_{j}^{n}(t)\right| d t\right) .
$$

By virtue of (3.3)-(3.6), we deduce that

$$
\begin{aligned}
& \mathbb{E}\left(\int_{0}^{T} \theta e^{\theta t}\left|Y_{i}^{m}(t)-Y_{i}^{n}(t)\right| d t\right)+\mathbb{E}\left(\int_{0}^{T}\left(e^{\theta t}-1\right)\left|D_{i}^{m}(t)-D_{i}^{n}(t)\right| d t\right) \\
\leq & \mathbb{E}\left(\int_{0}^{T}\left(e^{\theta t}-1\right)\left|b_{i}^{m}\left(t, Y^{m}(t)\right)-b_{i}^{n}\left(t, Y^{n}(t)\right)\right| 1_{A_{m, n}^{N}} d t\right) \\
+ & \mathbb{E}\left(\int_{0}^{T}\left(e^{\theta t}-1\right)\left|b_{i}^{m}\left(t, Y^{m}(t)\right)-b_{i}\left(t, Y^{m}(t)\right)\right| 1_{\bar{A}_{m, n}^{N}} d t\right) \\
+ & \mathbb{E}\left(\int_{0}^{T}\left(e^{\theta t}-1\right)\left|b_{i}\left(t, Y^{n}(t)\right)-b_{i}^{n}\left(t, Y^{n}(t)\right)\right| 1_{\bar{A}_{m, n}^{N}} d t\right) \\
+ & \left(\frac{L_{N}}{a_{i}}+L C_{1}\right) \mathbb{E}\left(\int_{0}^{T}\left(e^{\theta t}-1\right)\left\|Y^{m}(t)-Y^{n}(t)\right\| d t\right) \\
+ & \mathbb{E}\left(\int_{0}^{T}\left(e^{\theta t}-1\right) \sum_{j \neq i} v_{i j}\left|D_{j}^{m}(t)-D_{j}^{n}(t)\right| d t\right) .
\end{aligned}
$$


Multiplying (3.7) by $a_{i}$, adding and using $\sum_{i \neq j} a_{i} v_{i j} \leq \alpha a_{j}$, we obtain

$$
\begin{aligned}
& \theta d\left(\left(Y^{m}, K^{m}\right),\left(Y^{n}, K^{n}\right)\right) \\
\leq & \mathbb{E}\left(\sum_{i=1}^{d} \int_{0}^{T}\left(e^{\theta t}-1\right) a_{i}\left|b_{i}^{m}\left(t, Y^{m}(t)\right)-b_{i}^{n}\left(t, Y^{n}(t)\right)\right| 1_{A_{m, n}^{N}} d t\right) \\
+ & \mathbb{E}\left(\sum_{i=1}^{d} \int_{0}^{T}\left(e^{\theta t}-1\right) a_{i}\left|b_{i}^{m}\left(t, Y^{m}(t)\right)-b_{i}\left(t, Y^{m}(t)\right)\right| 1_{\bar{A}_{m, n}^{N}} d t\right) \\
+ & \mathbb{E}\left(\sum_{i=1}^{d} \int_{0}^{T}\left(e^{\theta t}-1\right) a_{i}\left|b_{i}\left(t, Y^{n}(t)\right)-b_{i}^{n}\left(t, Y^{n}(t)\right)\right| 1_{\bar{A}_{m, n}^{N}} d t\right) \\
& +\left(d L_{N}+\left(\sum_{i=1}^{d} a_{i}\right) L C_{1}\right) \mathbb{E}\left(\int_{0}^{T}\left(e^{\theta t}-1\right)\left\|Y^{m}(t)-Y^{n}(t)\right\| d t\right) \\
& +\alpha \mathbb{E}\left(\int_{0}^{T}\left(e^{\theta t}-1\right)\left\|D^{m}(t)-D^{n}(t)\right\| d t\right) .
\end{aligned}
$$

Choosing $\theta$ large enough such that $\frac{1}{\theta}\left(d L_{N}+\left(\sum_{i=1}^{d} a_{i}\right) L C_{1}\right) \leq \alpha$ leads to

$$
\begin{aligned}
d\left(\left(Y^{m}, K^{m}\right),\left(Y^{n}, K^{n}\right)\right) \leq & \alpha d\left(\left(Y^{m}, K^{m}\right),\left(Y^{n}, K^{n}\right)\right) \\
& +\frac{1}{\theta} \rho_{N}\left(b^{n}-b\right)+\frac{1}{\theta} \rho_{N}\left(b^{m}-b\right) \\
& +\frac{1}{\theta} C_{m, n}^{N}
\end{aligned}
$$

where

$$
\begin{aligned}
C_{m, n}^{N} & =\mathbb{E}\left(\sum_{i=1}^{d} \int_{0}^{T}\left(e^{\theta t}-1\right) a_{i}\left|b_{i}^{m}\left(t, Y^{m}(t)\right)-b_{i}^{n}\left(t, Y^{n}(t)\right)\right| 1_{A_{m, n}^{N}} d t\right) \\
& \leq 2 \sum_{i=1}^{d} \int_{0}^{T}\left(e^{\theta t}-1\right) a_{i} \beta_{i} \mathbb{E}\left(1_{A_{m, n}^{N}}\right) d t \\
& \leq \frac{2}{N} \sum_{i=1}^{d} a_{i} \beta_{i} \int_{0}^{T}\left(e^{\theta t}-1\right) \mathbb{E}\left(\left\|Y^{n}(t)\right\|+\left\|Y^{m}(t)\right\|\right) d t
\end{aligned}
$$

Let $C_{2}$ be such that

$$
\sum_{i=1}^{d} a_{i} \beta_{i}<C_{2}
$$

We have

$$
C_{m, n}^{N} \leq \frac{2 C_{2}}{N} \mathbb{E} \int_{0}^{T}\left(e^{\theta t}-1\right)\left(\left\|Y^{n}(t)\right\|+\left\|Y^{m}(t)\right\|\right) d t .
$$

By virtue of (3.1), there exists $C>0$ such that

$$
C_{m, n}^{N} \leq \frac{C}{N}
$$


Therefore

$$
(1-\alpha) d\left(\left(Y^{n}, K^{n}\right),\left(Y^{m}, K^{m}\right)\right) \leq \frac{1}{\theta}\left(\frac{C}{N}\right)+\frac{1}{\theta} \rho_{N}\left(b^{n}-b\right)+\frac{1}{\theta} \rho_{N}\left(b^{m}-b\right) .
$$

Passing to the limit on $n, m$ and $N$ in $(3.9)$, we deduce that $\left(Y^{n}, K^{n}\right)_{n \in \mathbb{N}}$ is a Cauchy sequence in $\widetilde{\mathcal{H}}$. Since $\widetilde{\mathcal{H}}$ is a Banach space, we set

$$
\lim _{n \rightarrow+\infty} Y^{n}=Y, \text { and } \lim _{n \rightarrow+\infty} K^{n}=K .
$$

If we return to the equation satisfied by the triple $\left(Y^{n}, K^{n}, Z^{n}\right)_{n \in \mathbb{N}}$ and use Itô's formula, we have

$$
\begin{aligned}
& \mathbb{E}\left(\left|Y_{i}^{m}(t)-Y_{i}^{n}(t)\right|^{2}\right)+\mathbb{E}\left(\int_{0}^{T} \sum_{j=1}^{d}\left|Z_{i j}^{m}(s)-Z_{i j}^{n}(s)\right|^{2} d s\right) \\
= & 2 \mathbb{E}\left(\int_{0}^{T}\left|Y_{i}^{m}(s)-Y_{i}^{n}(s)\right|\left|b_{i}^{m}\left(s, Y^{m}(s)\right)-b_{i}^{n}\left(s, Y^{n}(s)\right)\right| d s\right) \\
& +2 \mathbb{E}\left(\int_{0}^{T}\left|Y_{i}^{m}(s)-Y_{i}^{n}(s)\right|\left|D_{i}^{m}(s)-D_{i}^{n}(s)\right| d s\right) \\
& +2 \mathbb{E}\left(\int_{0}^{T}\left|Y_{i}^{m}(s)-Y_{i}^{n}(s)\right|\left|\sum_{j \neq i} r_{i j}\left(s, Y^{m}(s)\right) D_{j}^{m}(s)-r_{i j}\left(s, Y^{n}(s)\right) D_{j}^{n}(s)\right| d s\right) \\
\leq & 4 \beta_{i} \mathbb{E}\left(\int_{0}^{T}\left|Y_{i}^{m}(s)-Y_{i}^{n}(s)\right| d s\right)+4\left((I-V)^{-1} \beta\right)_{i} \mathbb{E}\left(\int_{0}^{T}\left|Y_{i}^{m}(s)-Y_{i}^{n}(s)\right| d s\right) \\
& +4 \sum_{j \neq i} v_{i j}\left((I-V)^{-1} \beta\right)_{j} \mathbb{E}\left(\int_{0}^{T}\left|Y_{i}^{m}(s)-Y_{i}^{n}(s)\right| d s\right) .
\end{aligned}
$$

Multiplying (3.10) by $a_{i}$ and adding leads to the existence of $C>0$ such that

$$
\begin{aligned}
& \mathbb{E}\left(\sum_{i=1}^{d} a_{i}\left|Y_{i}^{m}(t)-Y_{i}^{n}(t)\right|^{2}\right)+\mathbb{E}\left(\int_{0}^{T} \sum_{i=1}^{d} \sum_{j=1}^{d} a_{i}\left|Z_{i j}^{m}(s)-Z_{i j}^{n}(s)\right|^{2} d s\right) \\
\leq & 4 \mathbb{E}\left(\int_{0}^{T} \sum_{i=1}^{d} a_{i} \beta_{i}\left|Y_{i}^{m}(s)-Y_{i}^{n}(s)\right| d s\right) \\
& +4 \mathbb{E}\left(\int_{0}^{T} \sum_{i=1}^{d}\left((I-V)^{-1} \beta\right)_{i} a_{i}\left|Y_{i}^{m}(s)-Y_{i}^{n}(s)\right| d s\right) \\
& +4 \mathbb{E}\left(\int_{0}^{T} \sum_{i=1}^{d} \sum_{j \neq i} a_{i} v_{i j}\left((I-V)^{-1} \beta\right)_{j}\left|Y_{i}^{m}(s)-Y_{i}^{n}(s)\right| d s\right) . \\
\leq & C \mathbb{E}\left(\int_{0}^{T} \sum_{i=1}^{d} a_{i}\left|Y_{i}^{m}(s)-Y_{i}^{n}(s)\right| d s\right) .
\end{aligned}
$$


Passing to the limit on $m, n$, we deduce that $\left(Z^{n}\right)_{n \geq 1}$ is a Cauchy sequence in the Banach $\mathbb{H}$. Since $\mathbb{H}$ is a Banach space, we put

$$
Z=\lim _{n \rightarrow+\infty} Z^{n}
$$

Then

Lemma 3.3: Let $\left(Y^{n}, K^{n}, Z^{n}\right)_{n \geq 1}$ be the unique solution of the $R B S D E\left(\xi, b^{n}, R\right)$.

\section{Proof:Set}

$$
b^{n}\left(., Y^{n}\right) \text { converges to } b(., Y) \text { in }\left(L_{+}^{1}\left(\Omega \times[0, T], d \mathbb{P} \times e^{\theta t} d t\right)\right) \text {. }
$$

$$
A_{n}^{N}=\left\{\omega \in \Omega,\left\|Y^{n}(t, \omega)\right\|+\|Y(t, \omega)\|>N\right\}, \bar{A}_{n}^{N}=\Omega \backslash A .
$$

We have

$$
\begin{array}{r}
\mathbb{E}\left(\int_{0}^{T} e^{\theta t}\left|b_{i}^{n}\left(t, Y^{n}(t)\right)-b_{i}(t, Y(t))\right| d t\right) \\
\leq \mathbb{E}\left(\int_{0}^{T} e^{\theta t}\left|b_{i}^{n}\left(t, Y^{n}(t)\right)-b_{i}(t, Y(t))\right| 1_{A_{n}^{N}} d t\right) \\
+\mathbb{E}\left(\int_{0}^{T} e^{\theta t}\left|b_{i}^{n}\left(t, Y^{n}(t)\right)-b_{i}\left(t, Y^{n}(t)\right)\right| 1_{\bar{A}_{n}^{N}} d t\right) \\
+\mathbb{E}\left(\int_{0}^{T} e^{\theta t}\left|b_{i}\left(t, Y^{n}(t)\right)-b_{i}(t, Y(t))\right| 1_{\bar{A}_{n}^{N}} d t\right) \\
\leq \frac{2 \beta_{i}}{N} \mathbb{E}\left(\int_{0}^{T} e^{\theta t}\left(\left\|Y^{n}(t)\right\|+\|Y(t)\|\right) d t\right) \\
+\mathbb{E}\left(\int_{0}^{T} e^{\theta t}\left|b_{i}^{n}\left(t, Y^{n}(t)\right)-b_{i}\left(t, Y^{n}(t)\right)\right| 1_{\bar{A}_{n}^{N}} d t\right) \\
\frac{L_{N}}{a_{i}} \mathbb{E}\left(\int_{0}^{T} e^{\theta t}\left\|Y^{n}(t)-Y(t)\right\| d t\right) .
\end{array}
$$

Multiplying (3.12) by $a_{i}$ and adding, we get

$$
\begin{array}{r}
\mathbb{E}\left(\int_{0}^{T} e^{\theta t}\left\|b^{n}\left(t, Y^{n}(t)\right)-b(t, Y(t))\right\| d t\right) \\
\leq \rho_{N}\left(b^{n}-b\right)+\frac{2}{N} \sum_{i}^{d} \beta_{i} a_{i} \mathbb{E}\left(\int_{0}^{T} e^{\theta t}\left(\left\|Y^{n}(t)\right\|+\|Y(t)\|\right) d t\right) \\
+d L_{N} \mathbb{E}\left(\int_{0}^{T} e^{\theta t}\left\|Y^{n}(t)-Y(t)\right\| d t\right) .
\end{array}
$$

By virtue of (3.1), we deduce that there exists $C>0$ such that

$$
\begin{array}{r}
\mathbb{E}\left(\int_{0}^{T} e^{\theta t}\left\|b^{n}\left(t, Y^{n}(t)\right)-b(t, Y(t))\right\| d t\right) \\
\leq \rho_{N}\left(b^{n}-b\right)+\frac{C}{N}+d L_{N} \mathbb{E}\left(\int_{0}^{T} e^{\theta t}\left\|Y^{n}(t)-Y(t)\right\| d t\right) .
\end{array}
$$


Passing to the limit on $n, N$, completes the proof of Lemma 3.5.

Proof of Theorem 2.1:

Existence: Combining Lemmas (3.2)-(3.5) and passing to the limit in the RBSDE $\left(\xi, b^{n}, R\right)$, we deduce that the triple $\{(Y(t), K(t), Z(t)), 0 \leq t \leq T\}$ is a solution of our $\operatorname{RBSDE}(\xi, b, R)$.

Uniqueness: Let $\{(Y(t), K(t), Z(t)), 0 \leq t \leq T\}$ and $\left\{\left(Y^{\prime}(t), K^{\prime}(t), Z^{\prime}(t)\right), 0 \leq t \leq T\right\}$ be two solutions of our RBSDE. For every $t \geq 0$, define

$(\Delta Y(t), \Delta K(t), \Delta Z(t), \Delta D(t))=\left(Y(t)-Y^{\prime}(t), K(t)-K^{\prime}(t), Z(t)-Z^{\prime}(t), D(t)-D^{\prime}(t)\right)$.

We have

$$
\begin{aligned}
& \mathbb{E}\left(\int_{0}^{T} \theta e^{\theta t}\left|\Delta Y_{i}(t)\right| d t+\mathbb{E} \int_{0}^{T}\left(e^{\theta t}-1\right)\left|\Delta D_{i}(t)\right| d t\right) \\
\leq & \mathbb{E}\left(\int_{0}^{T}\left(e^{\theta t}-1\right)\left|b_{i}(t, Y(t))-b_{i}\left(t, Y^{\prime}(t)\right)\right| d t\right) \\
+ & \mathbb{E}\left(\int_{0}^{T}\left(e^{\theta t}-1\right) \sum_{j \neq i} \mid r_{i j}\left(t, Y(t)-r_{i j}\left(t, Y^{\prime}(t)\right)|| D_{j}(t) \mid d t\right)\right. \\
+ & \mathbb{E}\left(\int_{0}^{T}\left(e^{\theta t}-1\right) \sum_{j \neq i}\left|r_{i j}\left(t, Y^{\prime}(t)\right)\right|\left|\Delta D_{j}(t)\right| d t\right) .
\end{aligned}
$$

For an arbitrary number $N>1$, let $L_{N}$ be Lipschitz constant of $b$ in the ball $B(0, N)$. We put

$$
A^{N}=\left\{\omega \in \Omega,\|Y(t, \omega)\|+\left\|Y^{\prime}(t, \omega)\right\|>N\right\}, \bar{A}^{N}=\Omega \backslash A^{N} .
$$

By virtue of (3.13) and the Lipschitz continuity of $R$, we deduce that there exists $C_{1}>0$ such that

$$
\begin{aligned}
& \mathbb{E}\left(\int_{0}^{T} \theta e^{\theta t}\left|\Delta Y_{i}(t)\right| d t\right)+\mathbb{E}\left(\int_{0}^{T}\left(e^{\theta t}-1\right)\left|\Delta D_{i}(t)\right| d t\right) \\
\leq & \mathbb{E}\left(\int_{0}^{T}\left(e^{\theta t}-1\right)\left|b_{i}(t, Y(t))-b_{i}\left(t, Y^{\prime}(t)\right)\right| 1_{A^{N}} d t\right) \\
+ & \mathbb{E}\left(\int_{0}^{T}\left(e^{\theta t}-1\right)\left|b_{i}(t, Y(t))-b_{i}\left(t, Y^{\prime}(t)\right)\right| 1_{\bar{A}^{N}} d t\right) \\
+ & L C_{1} \mathbb{E}\left(\int_{0}^{T}\left(e^{\theta t}-1\right)\|\Delta Y(t)\| d t\right) \\
+ & \mathbb{E}\left(\int_{0}^{T}\left(e^{\theta t}-1\right) \sum_{j \neq i} v_{i j}\left|\Delta D_{j}(t)\right| d t\right) .
\end{aligned}
$$


From the boundeness condition on the coefficient $b$, we get

$$
\begin{aligned}
& \mathbb{E}\left(\int_{0}^{T} \theta e^{\theta t}\left|\Delta Y_{i}(t)\right| d t\right)+\mathbb{E}\left(\int_{0}^{T}\left(e^{\theta t}-1\right)\left|\Delta D_{i}(t)\right| d t\right) \\
\leq & \frac{2 \beta_{i}}{N} \mathbb{E}\left(\int_{0}^{T}\left(e^{\theta t}-1\right)\|Y(t)\|+\left\|Y^{\prime}(t)\right\| d t\right) \\
& +\left(L C_{1}+\frac{L_{N}}{a_{i}}\right) \mathbb{E}\left(\int_{0}^{T}\left(e^{\theta t}-1\|\Delta Z(t)\| d t\right)\right. \\
& +\mathbb{E} \int_{0}^{T}\left(e^{\theta t}-1\right) \sum_{j \neq i} v_{i j}\left|\Delta D_{j}(t)\right| d t .
\end{aligned}
$$

Multiplying (3.14) by $a_{i}$, adding and using (3.1) and the inequality $\sum_{i \neq j} a_{i} v_{i j} \leq \alpha a_{j}$, we get the existence of $C>0$ such that

$$
\begin{aligned}
\theta d\left((Y, Z),\left(Y^{\prime}, Z^{\prime}\right)\right) \leq & \frac{C}{N} \\
& +\left(d L_{N}+\left(\sum_{i=1}^{d} a_{i}\right) L C_{1}\right) \mathbb{E} \int_{0}^{T}\left(e^{\theta t}-1\right)\|\Delta Y(t)\| d t \\
& +\alpha \mathbb{E}\left(\int_{0}^{T}\left(e^{\theta t}-1\right)\|\Delta D(t)\| d t\right) .
\end{aligned}
$$

Choosing $\theta$ large enough such that $\frac{1}{\theta}\left(d L_{N}+\left(\sum_{i=1}^{d} a_{i}\right) L C_{1}\right) \leq \alpha$, we get

$$
d\left((Y, K),\left(Y^{\prime}, K^{\prime}\right)\right) \leq \frac{C}{\theta N}+\alpha d\left((Y, K),\left(Y^{\prime}, K^{\prime}\right)\right)
$$

Finally

$$
(1-\alpha) d\left((Y, K),\left(Y^{\prime}, K^{\prime}\right)\right) \leq \frac{C}{\theta N}
$$

which leads to

$$
Y=Y^{\prime} \text { and } K=K^{\prime}
$$

by letting $N$ going to $+\infty$.

By the same calculations as in (3.10) and (3.11), we obtain the existence of $C>0$ such that

$$
\begin{aligned}
& \mathbb{E}\left(\sum_{i=1}^{d} a_{i}\left|\Delta Y_{i}(t)\right|^{2}\right)+\mathbb{E}\left(\int_{0}^{T} \sum_{i=1}^{d} \sum_{j=1}^{d} a_{i}\left|\Delta Z_{i j}(s)\right|^{2} d s\right) \\
\leq & C \mathbb{E}\left(\int_{0}^{T} \sum_{i=1}^{d} a_{i}\left|\Delta Y_{i}(s)\right| d s\right) .
\end{aligned}
$$

Therefore

$$
Z=Z^{\prime}
$$




\section{References}

[1] Bismut, J.M., Conjugate convex function in optimal stochastic control, J. Math. Anal. Apl. 44 (1973), 384-404.

[2] Bismut, J.M., An introductory approch to duality in stochastic control, SIAM Rev. 20 (1978), 62-78.

[3] Cvitanic, J. and Karatzas, I. Backward stochastic differential equation with refection and Dynkin game, Annals Probab. 24:4 (1996), 2024-2056.

[4] Darling, R.W.R., Constructiing gamma martingales with prescribed limits, using backward SDEs, Annals Probab. 23 (1995), 1234-1261.

[5] El Karoui, N., Kapoudjian, C., Pardoux, E., Peng, S. and Quenez, M. C., Reflected solutions of backward SDE's, and related obstacle problems for PDE's, Annals Probab. 25:2 (1997), 702-737.

[6] El Karoui, N., Peng, S. and Quenez, M. C. Backward stochastic differential equation in finance, Math. Finance 7 (1997), 1-71.

[7] Essaky, E. H., Bahlali, K. and Ouknine, Y., Reflected backward stochastic differential equation with jumps and locally Lipschitz coefficient, Random Oper. Stoch. Eqs. 10:4 (2002), 335-350.

[8] Gegout-Petit, A. and Pardoux, E., Equations différentielles stochastiques retrogrades refléchies dans un convexe, Stoch. Rep. 57 (1996), 111-128.

[9] Hamadène, S. and Lepeltier, J. P., Zero-sum stochastic differential games and BSDEs, Sys. and Contr. Lett. 24 (1995), 259-263.

[10] Hamadène, S., Lepeltier, J. P. and Matoussi, A., Double barrierr reflected backward sde's with continuous coefficients, In: Backward Stoch. Differ. Eqs. Pitman Research Notes in Mathematic Series 364 (1997).

[11] Hamadène, S. and Ouknine, Y., Reflected backward stochastic differential equations with jumps and stochastic obstacle, Elect. J. Probab. 8:2 (2002), 1-20.

[12] Matoussi, A., Reflected solutions of BSDEs with continuous coefficient, Stat. and Probab. Lett. 34 (1997), 347-354.

[13] N'zi, M. and Ouknine, Y., Backward stochastic differential equations with jumps involving a subdifferential operator, Random Oper. Stoch. Equations 8:4 (2000), 305-414.

[14] Pardoux, E. and Peng, S., Backward stochastic differential equation and quasilinear parabolic partial differential equations, In: Stoch. Partial Equations and Their Applications (ed. by B.L. Rozovski and R.B. Sowers) Lect. Notes control Inf. Sci. 176 (1992), Springer, Berlin, 200-217.

[15] Pardoux, E. and Rascanu, A., Backward SDE's with maximal monotone operator, Stoch. Proc. Appl. 76:2 (1998), 191-215.

[16] Ramasubramanian, S., Reflected backward stochastic differential equations in an orthant, Proc. Indian. Acada. Sci. (Math. Sci.) 112:2 (2002), 347-360.

[17] Shashiahvili, M., The Skorohod oblique reflection problem in a convex polyhedron, Georgian Math. J 3 (1996), 153-176. 


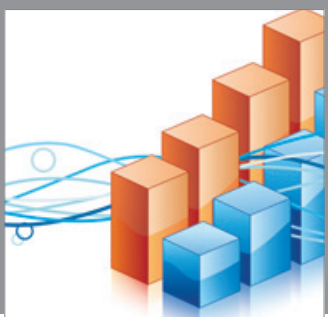

Advances in

Operations Research

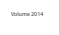

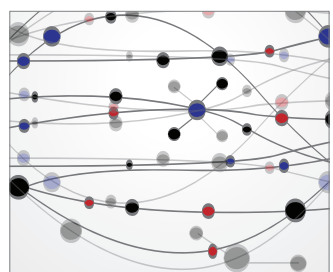

\section{The Scientific} World Journal
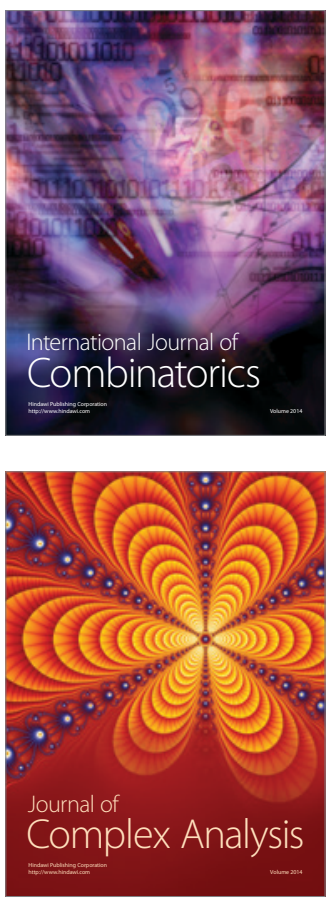

International Journal of

Mathematics and

Mathematical

Sciences
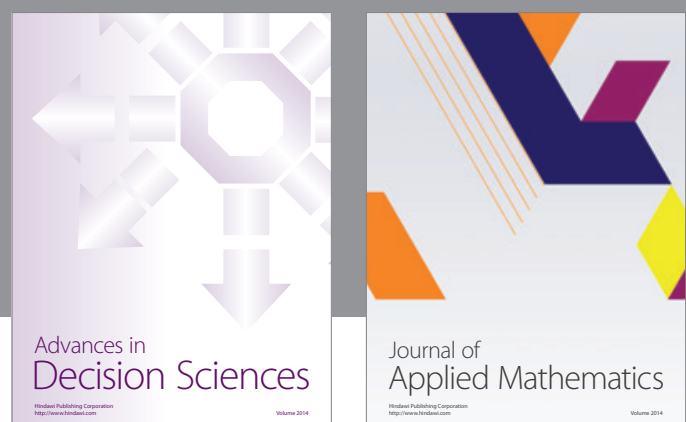

Journal of

Applied Mathematics
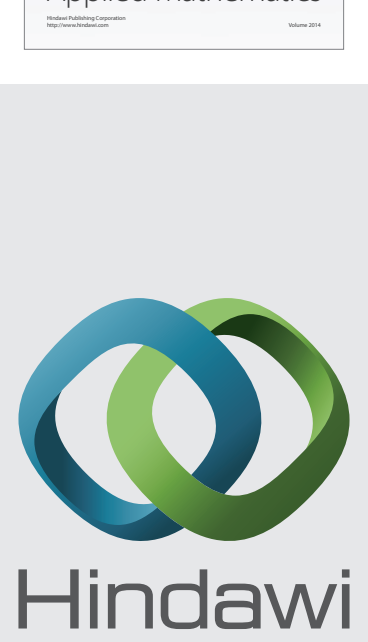

Submit your manuscripts at http://www.hindawi.com
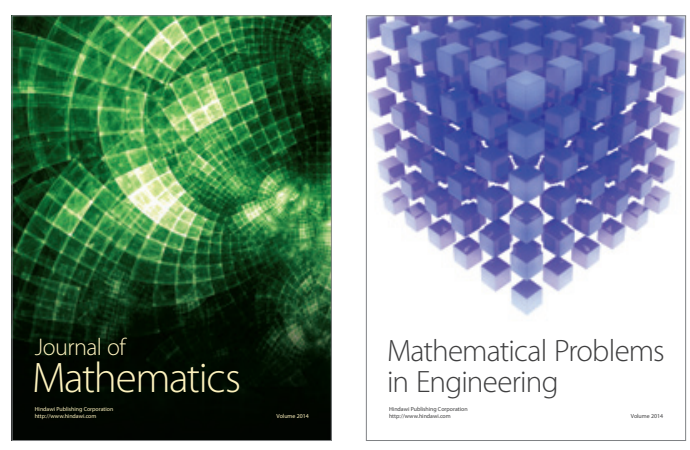

Mathematical Problems in Engineering
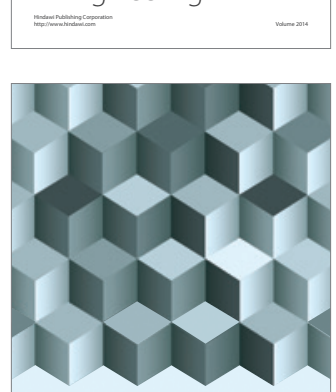

Journal of

Function Spaces
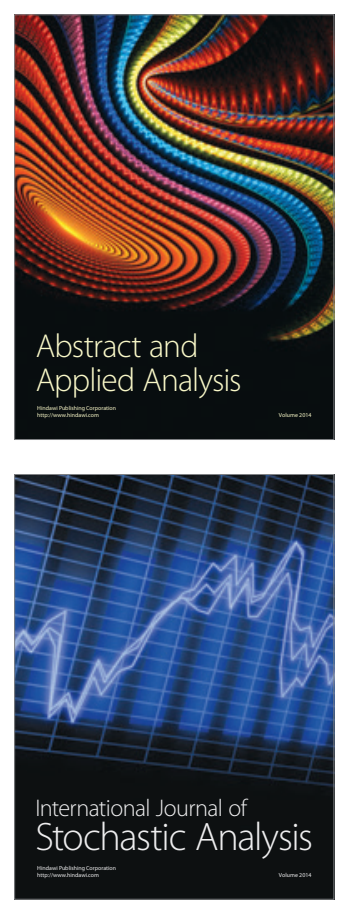

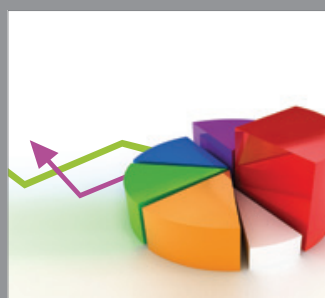

ournal of

Probability and Statistics

Promensencen
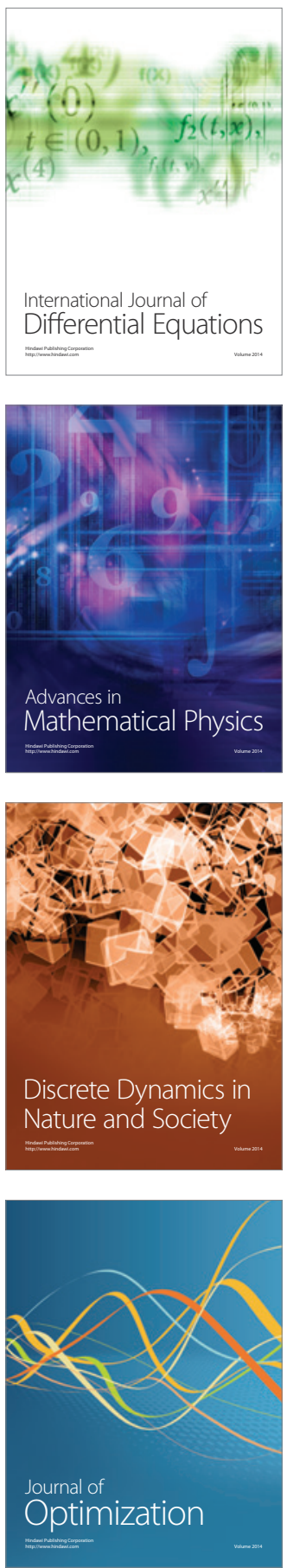